\title{
Optical Properties of Pentacene and Perfluoropentacene Thin Films
}

\author{
Alexander Hinderhofer, ${ }^{1}$ Ute Heinemeyer, ${ }^{1}$ Alexander Gerlach, ${ }^{1}$ Stefan Kowarik, ${ }^{1}$ \\ Robert M. J. Jacobs, ${ }^{2}$ Youichi Sakamoto, ${ }^{3}$ Toshiyasu Suzuki, ${ }^{3}$ and Frank Schreiber ${ }^{1, *}$ \\ ${ }^{1}$ Institut für Angewandte Physik Universität Tübingen, \\ Auf der Morgenstelle 10, 72076 Tübingen, Germany \\ ${ }^{2}$ Chemistry Research Laboratory, University of Oxford, Oxford OX1 3TA, U.K. \\ ${ }^{3}$ Institute for Molecular Science Myodaiji, Okazaki 444-8787, Japan
}

(Dated: October 31, 2018)

\begin{abstract}
The optical properties of pentacene $(\mathrm{PEN})$ and perfluoropentacene(PFP) thin films on various $\mathrm{SiO}_{2}$ substrates were studied using variable angle spectroscopic ellipsometry. Structural characterization was performed using X-ray reflectivity and atomic force microscopy. A uniaxial model with the optic axis normal to the sample surface was used to analyze the ellipsometry data. A Strong optical anisotropy was observed and enabled the direction of the transition dipole of the absorption bands to be determined. Furthermore, comparison of the optical constants of PEN and PFP thin films with the absorption spectra of the monomers in solution shows significant changes due to the crystalline environment. Relative to the monomer spectrum the HOMO-LUMO transition observed in PEN (PFP) thin film is reduced by $210 \mathrm{meV}(280 \mathrm{meV})$. Surprisingly, a second absorption band in the PFP thin film shows a slight blueshift $(40 \mathrm{meV})$ compared to the spectrum of the monomer with its transition dipole perpendicular to that of the first absorption band.
\end{abstract}

\section{INTRODUCTION}

One of the reasons for the substantial application potential of organic semiconducting molecules is their tunability, by exchanging certain functional groups of a molecule while leaving the backbone unchanged. For organic electronics in general and organic field effect transistors in particular pentacene $\left(\mathrm{C}_{22} \mathrm{H}_{14}, \mathrm{PEN}\right)$ is the most popular compound $\frac{122}{2}$, although there are many materials and compounds to choose from, and it is not entirely obvious why PEN would actually have to be the best choice. Possible alternatives to PEN may be, e.g., rubrene, which has its own structural subtleties ${ }^{3 / 415}$ and diindenoperylene, which exhibits excellent structural order $\frac{677889}{\text {. }}$.

Another option is to stay with the PEN backbone and study modifications of PEN. Recently, perfluorinated pentacene $\left(\mathrm{C}_{22} \mathrm{~F}_{14}, \mathrm{PFP}\right)$ has been identified as an interesting, possibly complementary option to PEN|10|11/12/13. Due to the strong electronegativity of the fluorine atoms the charge transport behavior changes from p-type into n-type, which opens the possibility of low-stress bipolar transistors. While there have been pioneering studies on PFP, its properties still require thorough investigation.

Even for PEN, despite the efforts in recent years, several issues regarding the growth, structure, and phase behavior are still under investigation $\frac{14 \mid 15 / 16 / 17}{}$. Thin films of pentacene on $\mathrm{SiO}_{2} \frac{16117}{1}$ and also on glass exhibit a coexistence of the 'thin film phase' and the 'bulk phase', the latter of which could be identified by a smaller $d(001)$ spacing. The substrate and its surface treatment influence the grain size and morphology, for example on glass the grain size is reported to be smaller than on oxidised silicon $18 \mid 19$.

Structural issues significantly impact the charge carrier mobility, which is the crucial quantity determining the frequency at which organic semiconductor devices can be operated. The optical properties in the condensed phase can, of course, also be strongly affected by the film structure because of the differences in the coupling of a given molecule with its environment, particularly for crystalline films of PEN and PFP. Due to the intimate relationship between the optical spectrum and electronic properties, efforts to improve our understanding of the material and the device performance have to include the optical properties, which in crystalline materials are generally anisotropic.

Recently, two studies focused on the optical properties of rather thick PEN films (100 and $44 \mathrm{~nm})^{20121}$ using isotropic models. The role of possible anisotropies as well as the behavior of thinner films and other growth regimes of PEN require further investigation. Specifically because thinner films exhibit a significant lower amount of the 'bulk phase' and are dominated by the 'thin film phase', these issues remain open.

This paper is devoted to a comparison of the optical properties of PFP and PEN (Fig. 1) thin films grown by organic molecular beam deposition $(\mathrm{OMBD})^{2223}$. This should serve, first, to establish a more solid data base for PEN and, second, to shed light on the properties of $\mathrm{PFP}$ as a rather new and promising material for organic electronics and optoelectronics.

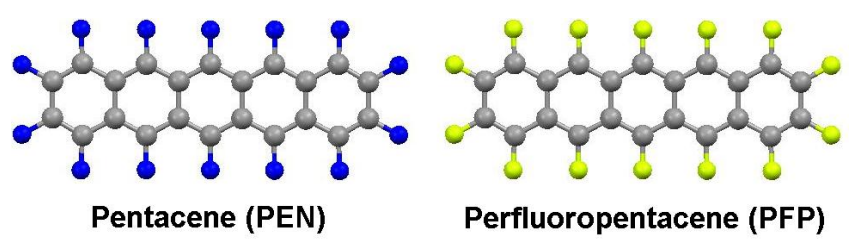

Figure 1: Scheme of the PEN $\left(\mathrm{C}_{22} \mathrm{H}_{14}\right)$ and PFP $\left(\mathrm{C}_{22} \mathrm{~F}_{14}\right)$ molecule. 


\section{EXPERIMENTAL}

Three different types of substrates were used, a $\sim 1 \mathrm{~mm}$ thick $\operatorname{Si}(100)$ wafer with a native oxide layer $(d \sim 1 \mathrm{~nm})$, a silicon wafer with a thermal $\mathrm{SiO}_{2}$ layer $(d=430 \mathrm{~nm})$ and a glass slide $(d=0.5 \mathrm{~mm})$. All substrates were cleaned with acetone and isopropanol in an ultrasonic bath and then rinsed with ultra pure water. The silicon oxide thickness (in the case of the Si wafers) and optical constants of the substrates were determined by ellipsometry prior to film growth. Organic molecular beam deposition (OMBD) under ultra high vacuum conditions (base pressure $2 \times 10^{-10}$ mbar) was used to grow PEN and PFP thin films by thermal evaporation from a Knudsen cell. The growth rate of about $2 \AA /$ min was monitored via a water-cooled quartz crystal microbalance and the substrate temperature was kept constant at $T=30^{\circ} \mathrm{C}$. Under these growth conditions PEN is structurally well ordered 14 .

The ellipsometry 24 data were taken ex-situ in air, within a few hours after growth, with a Woollam ellipsometer (M-2000, rotating compensator type) in an energy range from $1.25-3.5 \mathrm{eV}$. The spectra were recorded by a CCD camera with a wavelength resolution of $1.6 \mathrm{~nm}$. Two different types of measurements were performed, namely reflection and transmission ellipsometry. For reflection ellipsometry an automated goniometer was used to record data at 13 different angles of incidences between $40^{\circ}$ and $80^{\circ}$. In transmission we measured at five different angles between $20^{\circ}$ and $70^{\circ}$, manually aligned with an error of $\pm 0.5^{\circ}$. The data were analyzed based on established routines using a commercial software (WVASE32) $\sqrt{25 \mid 26}$. In-situ X-ray reflectivity measurements $(\lambda=0.92 \AA)$ were performed similar to Ref. 6] in UHV at beamline ID10B of the ESRF in Grenoble, France. Details will be published in Ref. 27.

Absorption spectra of PEN and PFP were recorded with a Varian Cary50 UV-VIS spectrometer. For this PEN and PFP material were dissolved at low concentration in dichlorobenzene and measured immediately in order to avoid dissociation effects which could be observed after several hours. Tapping mode AFM-measurements were performed in air about two to four months after deposition using a Digital Instruments Multimode AFM.

\section{RESULTS}

\section{A. Structure and Morphology}

As has been demonstrated before PEN $\sqrt{15}$ and PFP grow in crystalline films. The film structure was studied by X-ray reflectivity and AFM, as complementary techniques, with the purpose of supporting and complementing the optical spectra obtained by spectroscopic ellipsometry. Out-of-plane X-ray measurements of the first three Bragg reflections of PEN and PFP are shown in Fig. 2. The pronounced Bragg reflections with Laue oscillations and the width of the rocking curve at the first Bragg reflection of PFP $\left(0.0089^{\circ}\right)$ demonstrate the high structural order of the thin films. From the position of the second and third Bragg reflections the out-of-plane $d(001)$ lattice spacing is determined to be $15.4 \AA$ in PEN and $15.7 \AA$ in PFP, in agreement with the PFP thin film $\mathrm{X}$-ray data from Ref. 12 .

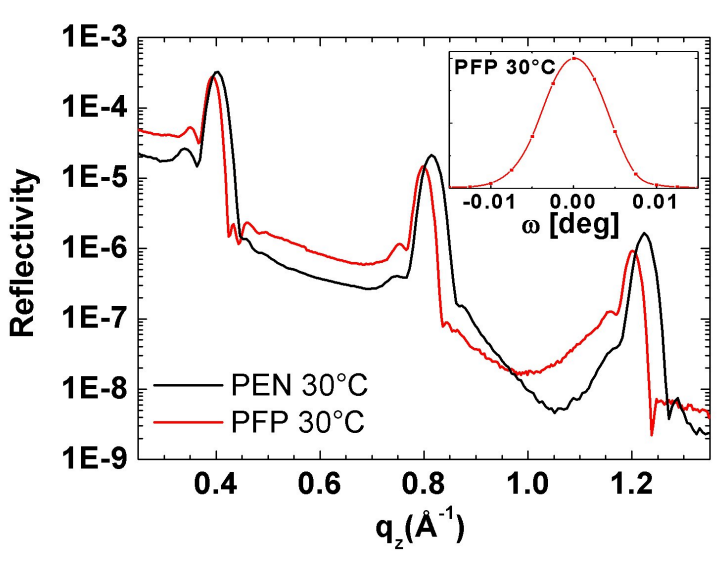

Figure 2: X-ray reflectivity data for $\operatorname{PEN}(d=16 \mathrm{~nm})$ and $\operatorname{PFP}(d=25 \mathrm{~nm})$ grown at $30^{\circ} \mathrm{C}$ substrate temperature on a silicon wafer with native oxide. The inset shows the narrow rocking width at the first Bragg reflection of PFP.

Both for PFP and PEN in the specular reflectivity in Fig. 2 Bragg reflections corresponding to only one polymorph are observed. For PEN though grazing incidence X-ray diffraction (GIXD) reveals small traces of a second phase. Apart from this issue of phase coexistence in PEN, the structural motif appears to be rather similar for PFP and PEN for growth at $T=30^{\circ} \mathrm{C}$.

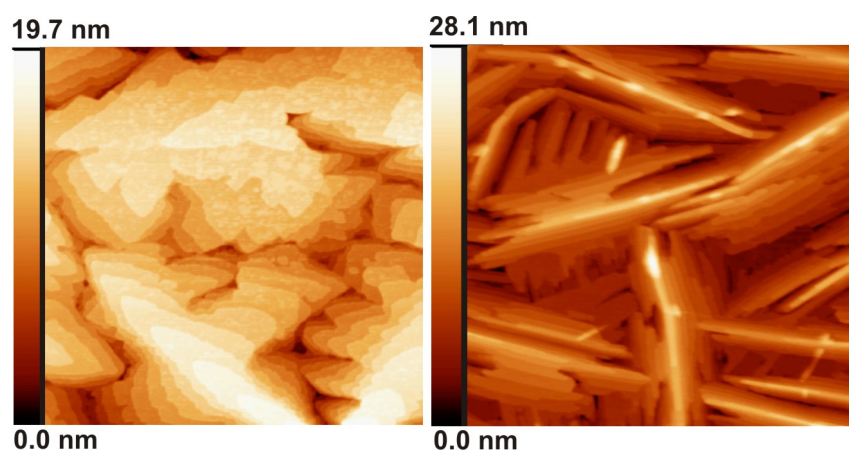

Figure 3: Typical morphologies for PEN and PFP thin films grown at $30{ }^{\circ} \mathrm{C}$ on native $\mathrm{SiO}_{2}$ at $22.5 \mathrm{~nm}$ thickness. Each picture shows an area of $3 \mu \mathrm{m} \times 3 \mu \mathrm{m}$. Left: Large grains of PEN. Right: PFP grows in lamellar shaped grains.

AFM data from PEN and PFP (Fig. 3) show the morphology of the thin films on native $\mathrm{SiO}_{2}$. Both thin films consist of randomly oriented crystalline grains. Steps between monolayers are observable with a typical height of 
$1.6-2.2 \mathrm{~nm}$ for PEN and $1.6-1.9 \mathrm{~nm}$ for PFP. These almost correspond to the $d(001)$ spacing as determined from X-ray measurements. Obviously, on isotropic substrates such as oxidized silicon, there is no azimuthal order, which results in an in-plane isotropic film structure.

\section{B. Optical properties}

Due to the high out-of-plane order and the in-plane isotropy both systems exhibit uniaxial optical properties with the optic axis perpendicular to the substrate surface. The determination of the optical constants for this geometry is a widely discussed issue in ellipsometry, see e.g. Ref. 28, Generally, an isotropic fit approach will not give the average properties of both axes ${ }^{29}$ but will produce artificial absorption feature ${ }^{30}$.

For the samples presented here (silicon substrate with native oxide and a $\sim 20 \mathrm{~nm}$ thin uniaxial film on top) standard Variable Angle Spectroscopic Ellipsometry (VASE) is not sensitive enough for determining a unique set of four optical constants, i.e. real and imaginary part of the in-plane and out-of-plane component at each wavelength 29131 . Therefore we used two techniques to increase the sensitivity to the anisotropy of our samples. In both cases we combined data from a thin film grown on native oxide with data from a simultaneously grown film on another substrate to perform a multisample analysis. First, using a substrate with a thick thermal oxide $(430 \mathrm{~nm})$ increases the sensitivity, because the intermediate layer decorrelates data measured at different angles of incidence ${ }^{31}$. The drawback of this method is that the exact determination of the thermal oxide optical constants is crucial for the analysis of organic thin films. Therefore it is useful to compare the results with an independent second approach where reflection data are combined with complementary transmission ellipsometry data from a glass sample ${ }^{\sqrt{32}}$. In this case, the thickness of the 'roughness layer' within the ellipsometric model must be adjusted accounting for the higher film roughness on glass.

Our layer model for the ellipsometry data is from bottom to top: substrate ([silicon + oxide] or glass) // uniaxial thin film // Effective Medium Approximation EMA (mixing void and uniaxial thin film 50\%). The thicknesses of the EMA layers are defined by AFMmeasurements, i.e. $8.5 \mathrm{~nm}$ for PEN and $10 \mathrm{~nm}$ for PFP for the samples studied. The AFM images show that the thin films have structures on the length scale of the wavelength of light used for ellipsometry, for this case an EMA model usually gives only an approximate representation of the morphology. The effective thicknesses of the thin films are determined isotropically in the transparent range by parameterization of the refractive index dispersion with a Cauchy function $(22.5 \mathrm{~nm})$. The uniaxial results obtained with both multisample approaches agree with each other, both for PEN as well as for PFP thin films. Thus we are able to determine the direction of the transition dipole $\vec{P}=\left(p_{\perp}, p_{\|}\right)$for each band.
We do not observe significant differences between ellipsometry data measured in-situ under UHV conditions and ellipsometry data measured ex-situ in air, hence we can exclude strong oxidation effects as they occur for instance in rubrene ${ }^{26}$.

\section{Comparison of PFP and PEN thin films.}

Fig. 4 displays the spectra as obtained with the above multisample approach for PEN and PFP thin films, respectively. In the energy range of $1.5-2.6 \mathrm{eV}$ we concentrate in the following on the in-plane component, since this is the dominating contribution and under the given experimental conditions the orthogonal out-ofplane component is very weak. The latter is at least five times smaller than the in-plane component and thus difficult to resolve reliably (relative error $50 \%$ ). In this
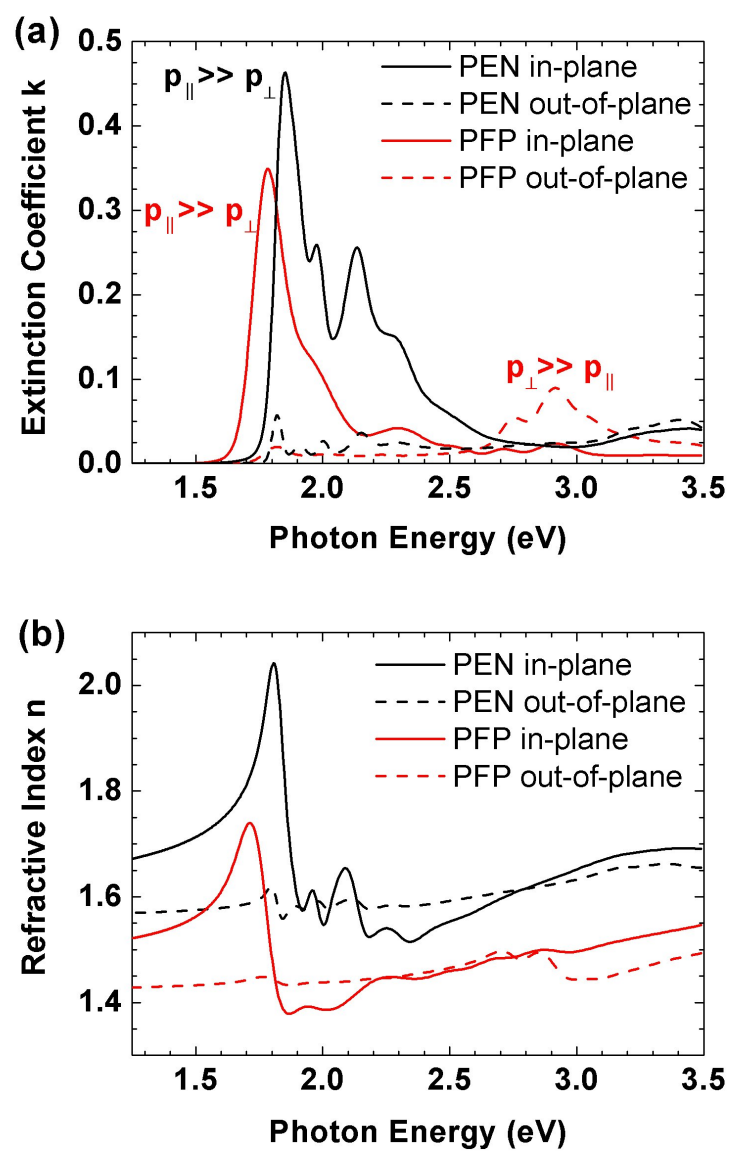

Figure 4: Optical constants for the in-plane and out-of-plane components of $22.5 \mathrm{~nm} \mathrm{PEN} \mathrm{and} 22.5 \mathrm{~nm}$ PFP thin films grown at $30^{\circ} \mathrm{C}$ substrate temperature, obtained by multisample analysis. (a) Extinction coefficients. (b) Refractive indices.

energy range the out-of-plane component is probably a small projection of the absorption band with the transition dipole along the short axis of the PEN and PFP 
molecules on the surface normal $\left(p_{\|} \gg p_{\perp}\right)$. This is also in good agreement with the tilt angle of pentacene molecules on $\mathrm{SiO}_{2}$, i.e. $10 \pm 5^{\circ}$ with respect to the surface normal, and with the idea that the transition dipole is essentially perpendicular to the long axis of the molecule 33 . At energies above $2.6 \mathrm{eV}$ the out-of-plane component exceeds the in-plane component in case of PFP and is thus well defined.

The error for the extinction coefficient $k$ peak positions is $\pm 0.01 \mathrm{eV}$, the error for the absolute values of $k$ is \pm 0.05 for both PEN and PFP. The absolute error for $n_{\infty}$ is approximately \pm 0.05 for all four determined refractive indices. The refractive index shown in Fig. 4 is KramersKronig consistent with $k$.

In Fig. 4 the PEN spectrum shows a pronounced first peak whose energy position of $1.85 \mathrm{eV}$ indicates the optical band gap in agreement with absorption measurements on glass and previous ellipsometry measurements $20 \mid 34$ The spectral feature at $1.97 \mathrm{eV}$ has been assigned to Davydov-splitting $34|35| 36$ but is currently still debated 37 . The subsequent peaks above $2 \mathrm{eV}$ exhibit an equal energy spacing of $170 \pm 10 \mathrm{meV}$ and are therefore likely to be associated with a vibronic progression.

The effect of substituting all hydrogen atoms by fluorine atoms results in interesting changes of the extinction coefficient. The optical band gap is red-shifted by $70 \mathrm{meV}$ in PFP compared to PEN. While the shift of the second peak $(\sim 1.94 \mathrm{eV})$ in $\mathrm{PFP}$ with only $\sim 30 \mathrm{meV}$ is smaller, it is much broader and appears therefore as a shoulder of the first peak. Interestingly, the third peak of the PEN spectrum is not visible in the PFP spectrum and only the fourth $(2.30 \mathrm{eV})$ and fifth $(2.48 \mathrm{eV})$ feature can be observed with similar energy spacing and position as in PEN. Another significant difference between PEN and PFP thin film spectra can be observed at energies above $2.6 \mathrm{eV}$. While for PEN there is no further absorption band visible in either component, new features appear for PFP. They can be found in both components, but the extinction coefficient is about four times bigger in the out-of-plane component showing that the transition dipole lies approximately perpendicular to the sample surface $\left(p_{\perp} \gg p_{\|}\right)$, i.e. along the long axis of the molecule, in contrast to the absorption band at lower energies.

\section{Solvent spectra of PEN and PFP}

Fig. 5 shows the absorption spectra of PEN and PFP in dichlorobenzene. Since the absolute values are not obtained by this measurement, the spectra for PFP and $\mathrm{PEN}$ are normalized to detect changes in relative amplitudes between both molecules. Our measurements are in agreement with Ref. 10. In the PFP solvent spectra two absorption bands with a vibronic progression (energy spacing $170 \pm 10 \mathrm{meV}$ ) are observed. The second band starting at $2.71 \mathrm{eV}$ (assigned to the $S_{0} \rightarrow S_{3}$ transition in Ref. (38) is more intense than the first at $1.99 \mathrm{eV}$
$\left(S_{0} \rightarrow S_{1}\right)$. In contrast, the $S_{0} \rightarrow S_{1}$ absorption band of PEN at $2.13 \mathrm{eV}$ is much more intense than the $S_{0} \rightarrow S_{3}$ band at $\sim 2.85 \mathrm{eV}$. The energy spacing of the first absorption band is similar to that of PFP, which implies that the vibronic progression for both molecules depends mainly on the vibrations of the carbon core. This is reasonable since the lengths of the $\mathrm{C}-\mathrm{C}$ bonds change significantly compared to the lengths of the $\mathrm{C}-\mathrm{H}$ bonds for a HOMOLUMO transition of PEN2, i.e. the HOMO-LUMO transition couples more strongly to the carbon core. Obviously, the fluorination changes the resulting oscillator strengths of the absorption bands substantially, which is reproduced by time-dependent density functional theory calculations 38 .

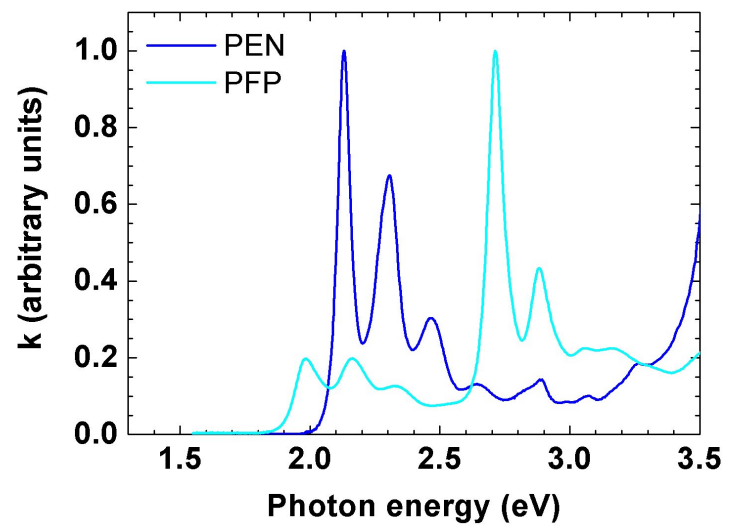

Figure 5: Normalized absorption spectra of PEN and PFP dissolved in dichlorobenzene.

\section{Comparison of thin films and solution spectra}

The comparison of the crystalline thin film spectra to the spectra of the monomer in solution gives information about the changes of the spectra induced by the crystal structure and the coupling of the molecules. This is shown in Fig. 6. For both molecules in solution one can clearly distinguish the vibronic transitions, whereas in the thin film all features are smeared out. For both materials the HOMO-LUMO absorption band in thin films is shifted to lower energies with respect to the monomer spectrum, i.e. $210 \mathrm{meV}$ in case of $\mathrm{PFP}$, and $280 \mathrm{meV}$ in case of PEN. The second absorption band shifts slightly to the blue $(40 \mathrm{meV}$ ) for PFP, and is not observable for $\mathrm{PEN}$. The beginning of another weak band in the range of $3.25-3.5 \mathrm{eV}$ for PEN corresponds to the tail of the strong $\beta$-band in solution 39 .

In both the intensity distributions of the first absorption band of PEN and PFP a strong split feature is observed at low energy in contrast to the solution spectra. For the second absorption band of PFP the inten- 
sity maximum shifts from the first to the second vibronic excitation.
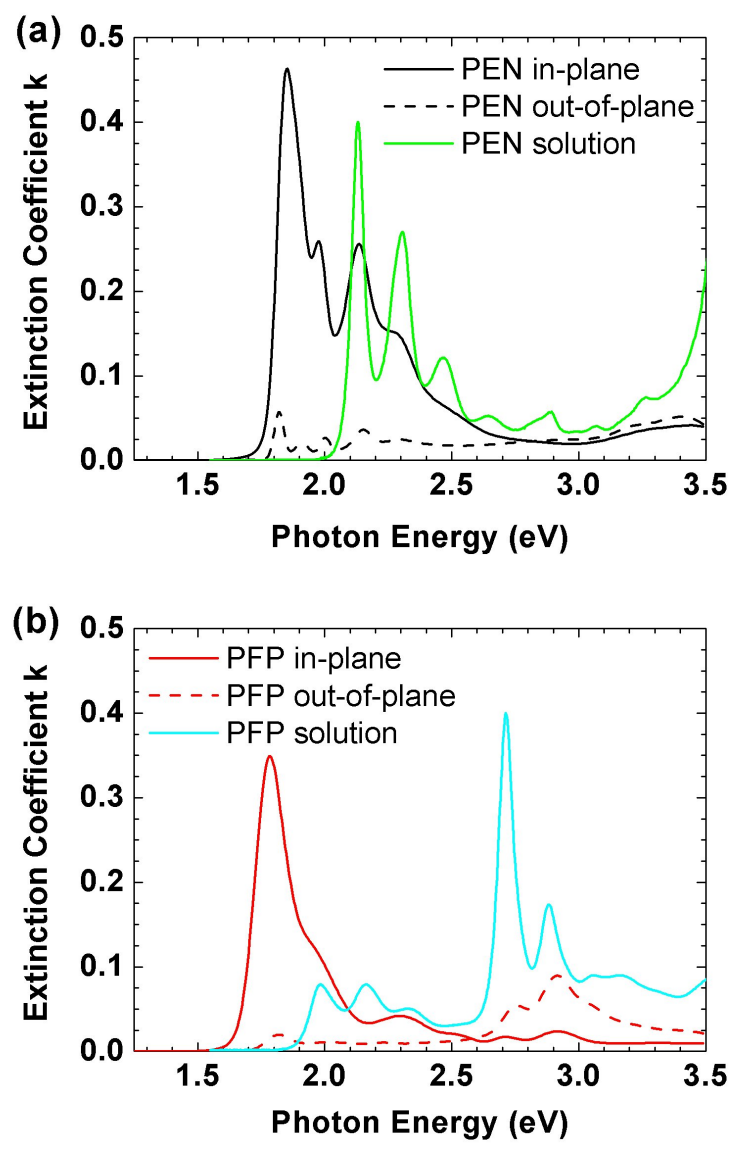

Figure 6: Thin film spectra of $22.5 \mathrm{~nm}$ thick films, growth temperature $30^{\circ} \mathrm{C}$ and spectra in solution. The intensity for the spectrum in solution is given in arbitrary units. a) PEN b) PFP: Note that around $3 \mathrm{eV}$ the out-of-plane component can be determined reliably, since it is actually rather strong in this energy range.

\section{DISCUSSION}

PEN thin films grown at $30^{\circ} \mathrm{C}$ on $\mathrm{Si}$ substrates have a $d(001)$-spacing of $15.4 \AA$, for which the molecular arrangement within the unit cell is still not established. Tiago et al $\stackrel{40}{ }$ calculated optical constants for two phases of crystalline PEN with a $d(001)$-spacing of $14.1 \AA$ and $14.4 \AA$, respectively. The calculated optical spectra of both phases show significant differences to each other with regard to absolute values and peak positions, a quantitative comparison with the $15.4 \AA$ 'thin film' phase may therefore be problematic.

Nevertheless, the calculated spectra for both phases show strong excitation at low energies with transition dipoles along the crystallographic a- and b-axis (overlap of $\pi$-bonds) with an excitonic character and weak excitation with a transition dipole along the crystallographic c-axis.

This is also valid for the 'thin film phase' we investigate in this paper; the a- and b-axis of the thin film crystallites coincide with the measured in-plane component while the out-of-plane component corresponds nearly to the crystallographic c-axis. In agreement with calculations from Ref. 40 the measured out-of-plane component exhibits a very weak transition dipole, whereas the in-plane component shows a strong first absorption feature. The pronounced red-shift of this in-plane absorption band in the thin film spectra relative to the monomer can thus be attributed to the generation of excitons.

Due to the observed structural and optical similarities of PEN and PFP one may speculate that for PFP the same excitonic behavior is present. Then the change in the first absorption band can also be explained by strong exciton generation. The behavior of the second band, with a transition dipole nearly perpendicular to the first band and which corresponds to the $S_{0} \rightarrow S_{3}$ transition of the monomer is different, its excitation energies are nearly the same as those for the monomer apart from a small solvent shift to the blue $(40 \mathrm{meV})$.

\section{SUMMARY AND CONCLUSION}

In conclusion, we have provided a comparison of PEN and PFP thin films. Structurally, the two materials follow similar motifs, although quantitatively slightly different with regard to the $d(001)$ lattice spacing and the morphology.

The spectra for PEN and PFP thin films were presented and compared to each other. A comparison of our results for the PEN 'thin film phase' with those obtained by Faltermeier et al! ${ }^{20}$ using an effective isotropic fit shows quantitative agreement regarding peak positions, but differ in relative and absolute intensities. We used an alternative approach by modeling the 'thin film phase' of PEN anisotropically, with the optic axis normal to the sample surface (out-of-plane component) and isotropic properties in the sample plane (in-plane component). With this approach we can determine the directions of the transition dipoles for each absorption band, which for the first band in PEN is approximately perpendicular to the surface normal. Significant differences were detected for the spectrum of the thin film compared to those of the monomer. These changes can be explained by strong exciton generation calculated by Tiago et al.

Two absorption bands were found in PFP thin films, the first has a transition dipole approximately perpendicular to the surface normal and shows a strong redshift in respect to the spectrum of the monomer, which is very similar to the behavior of the first absorption band of PEN. The transition dipole of the second band however lies nearly parallel to the surface normal. For this band the molecular states remain intact apart from a slight 
blue shift.

Given these results one observes that coupling between molecules in the condensed phase for PEN and PFP leads to strong effects on the optical spectra. This may be due to exciton generation in the plane where strong $\pi$-overlap occurs, but the molecular states with a transition dipole perpendicular to this plane remain nearly unchanged.

\section{ACKNOWLEDGMENTS}

Financial support by the EPSRC and the University of Tübingen is gratefully acknowledged. We thank Kanto Denka Kogyo Co., Ltd. for providing the PFP.
* Electronic address: frank.schreiber@uni-tuebingen.de

1 W. Brütting, Physics of Organic Semiconductors (WileyVCH, Weinheim, 2005).

2 R. Scholz, "Organic Semiconductors" in Encyclopedia of Condensed Matter Physics (edited by G. Bassani, G. Liedl, P. Wyder; Elsevier, 2005).

3 D. Käfer, L. Ruppel, G. Witte, and C. Wöll, Phys. Rev. Lett. 95, 166602 (2005).

4 S. Kowarik, A. Gerlach, S. Sellner, F. Schreiber, J. Pflaum, L. Cavalcanti, and O. Konovalov, Phys. Chem. Chem. Phys. 8, 1834 (2006).

${ }^{5}$ V. C. Sundar, J. Zaumseil, V. Podzorov, E. Menard, R. L. Willett, T. Someya, M. E. Gershenson, and J. A. Rogers, Science 303, 1644 (2004).

6 S. Kowarik, A. Gerlach, S. Sellner, F. Schreiber, L. Cavalcanti, and O. Konovalov, Phys. Rev. Lett. 96, 125504 (2006).

7 A. C. Dürr, F. Schreiber, M. Münch, N. Karl, B. Krause, V. Kruppa, and H. Dosch, Appl. Phys. Lett. 81, 2276 (2002).

8 A. Tripathi and J. Pflaum, Appl. Phys. Lett. 89, 082103 (2006).

9 N. Karl, in Organic electronic materials (edited by R. Farchioni and G. Grosso; Springer, Berlin, 2001).

10 Y. Sakamoto, T. Suzuki, M. Kobayashi, Y. Gao, Y. Fukai, Y. Inoue, F. Sato, and S. Tokito, J. Am. Chem. Soc. 126, 8138 (2004).

11 Y. Sakamoto, T. Suzuki, M. Kobayashi, Y. Gao, Y. Inoue, and S. Tokito, Mol. Crys. Liq. Crys. 444, 225 (2006).

12 Y. Inoue, Y. Sakamoto, T. Suzuki, M. Kobayashi, Y. Gao, and S. Tokito, Jap. J. Appl. Phys. 44, 3663 (2005).

13 N. Koch, A. Vollmer, S. Duhm, Y. Sakamoto, and T. Suzuki, Adv. Mater. 19, 112 (2007).

14 R. Ruiz, D. Choudhary, B. Nickel, T. Toccoli, K. Chang, A. Mayer, P. Clancy, J. M. Blakely, R. L. Headrick, S. Iannotta, et al., Chem. Mater. 16, 4497 (2004).

15 S. Kowarik, A. Gerlach, W. Leitenberger, J. Hu, G. Witte, C. Wöll, U. Pietsch, and F. Schreiber, Thin Solid Films 515, 5606 (2007).

16 I. P. M. Bouchoms, W. A. Schoonveld, J. Vrijmoeth, and T. Klapwijk, Synth. Met. 104, 175 (1999).

17 A. C. Mayer, A. Kazimirov, and G. G. Malliaras, Phys. Rev. Lett. 97, 105503 (2006).

18 J. Puigdollers, C. Voz, A. Orpella, I. Martin, M. Vetter, and R. Alcubilla, Thin Solid Films 427, 367 (2003).

19 C. Kim, K. Bang, I. An, C. J. Kang, Y. S. Kim, and D. Jeon, Curr. Appl. Phys. 6, 925 (2005).

20 D. Faltermeier, B. Gompf, M. Dressel, A. K. Tripathi, and J. Pflaum, Phys. Rev. B 74, 125416 (2006).

21 S. P. Park, S. S. Kim, J. H. Kim, C. N. Whang, and S. Ima, Appl. Phys. Lett. 80, 2872 (2002).

22 F. Schreiber, Phys. Stat. Sol. (a) 201, 1037 (2004).

23 G. Witte and C. Wöll, J. Mater. Res. 19, 1889 (2004).

24 R.M.A. Azzam and N.M. Bashara, Ellipsometry and Polarized Light (North-Holland Publishing Company, Amsterdam New York Oxford, 1977).

25 J.A. Woolam Co., WVASE32 Manual (2005).

26 M. Kytka, A. Gerlach, F. Schreiber, and J. Kováč, Appl. Phys. Lett. 90, 131911 (2007).

$27 \mathrm{~S}$. Kowarik et al. (in preparation).

28 M. Campoy-Quiles, P. G. Etchegoin, and D. D. C. Bradley, Phys. Rev. B 72, 045209 (2005).

29 D. J. D. Smet, J. Appl. Phys. 76, 2571 (1994).

30 M. J. Dignam, M. Moskovits, and R. W. Stobie, Trans. Farad. Soc. 67, 3306 (1971).

31 E. G. Bortchagovsky, Thin Solid Films 307, 192 (1997).

32 C. M. Ramsdale and N. C. Greenham, Adv. Mater. 14, 212 (2002).

33 G. Yoshikawa, T. Miyadera, R. Onoki, K. Ueno, I. Nakai, S. Entani, S. Ikeda, D. Guo, M. Kiguchi, H. Kondoh, et al., Surface Science 600, 2518 (2006).

34 O. Ostroverkhova, S. Shcherbyna, D. G. Cooke, R. F. Egerton, R. R. Tykwinski, S. R. Parkin, and J. E. Anthony, J. Appl. Phys. 98, 033701 (2005).

35 R. Hesse, W. Hofberger, and H. Baessler, Chem. Phys. 49, 201 (1980).

${ }^{36}$ K. O. Lee and T. T. Gan, Chem. Phys. Lett. 51, 120 (1977).

37 M. Grobosch, R. Schuster, T. Pichler, and M. Knupfer, Phys. Rev. B 74, 155202 (2006).

38 B. M. Medina, D. Beljonne, H. Egelhaaf, and J. Gierschner, J. Chem. Phys. 126, 111101 (2007).

39 J. B. Birks, Photophysics of Aromatic Molecules (Wiley, 1970).

40 M. L. Tiago, J. E. Northrup, and S. G. Louie, Phys Rev. B 67, 115212 (2003). 